\title{
REABILITAÇÃO SENSÓRIO-MOTORA ASSOCIADA À FOTOBIOMODULAÇÃO TRANSCRANIANA EM PACIENTES COM LESÃO DO SISTEMA NERVOSO CENTRAL
}

\section{SENSORY MOTOR REHABILITATION ASSOCIATED WITH TRANSCRANIAL PHOTOBIOMODULATION IN PATIENTS WITH INJURE IN THE CENTRAL NERVOUS SYSTEM}

\author{
Carolina Lobo Guimarães ${ }^{1}$ \\ Ana Paula Pinto ${ }^{2}$ \\ Humberto Gimenes Macedo ${ }^{3}$ \\ Virgínia Klausner ${ }^{4}$ \\ Fernanda Púpio Silva Lima ${ }^{5}$ \\ Rodrigo Alvaro Brandão Lopes Martins ${ }^{6}$ \\ Mário Oliveira Lima ${ }^{7}$
}

\begin{abstract}
Resumo: As doenças neurológicas geralmente estão associadas a importantes déficits funcionais, dificultando a execução de atividades de vida diária e autocuidado. Pesquisas recentes demonstram efeitos positivos da Fotobiomodulação Transcraniana (FBM-T) para estimulação do tecido cerebral. O estudo teve como objetivo avaliar os efeitos da FBM-T associada ao treinamento aeróbico na função do sistema musculoesquelético de indivíduos com lesões neurológicas crônicas. Participaram deste ensaio clínico duplo-cego 16 voluntários com diferentes lesões do SNC, de ambos os sexos, idade média de 51,9 $\pm 13,9$ anos e marcha preservada. Os voluntários foram divididos aleatoriamente em 2 grupos (Treino FBM-T ativo + treino aeróbico e FBM-T placebo + treino aeróbico). O programa de reabilitação foi realizado em esteira ergométrica, na frequência de 2 vezes semanais e com duração total de 12 semanas. A FBM-T (3 lasers no $\lambda=660 \mathrm{~nm}$ e 3 Lasers no $\lambda=808 \mathrm{~nm}, 100 \mathrm{~mW}$, $6 \mathrm{~J} /$ laser, $60 \mathrm{seg} /$ ponto, $36 \mathrm{~J}$ por ponto, energia total $108 \mathrm{~J} / \mathrm{cm} 2$ ) foi aplicada em 3 pontos, sendo estes Fz, F7 e F8 segundo o Sistema Internacional 10-20 de EEG. Foram avaliados RMS do músculo reto femoral parético ou de maior comprometimento no pré-tratamento, $8^{a}$ semana e $12^{a}$ semana. Nota-se um aumento da atividade elétrica do grupo Laser nas coletas de RMS isotônica e isométrica, e redução do recrutamento muscular em ortostatismo quando comparados o início e o final do treinamento. Sugere-se que a FBM-T pode ter sido um fator influente para o aumento do recrutamento muscular no grupo Laser.
\end{abstract}

\footnotetext{
1 Mestre em Engenharia Biomédica, Universidade do Vale do Paraíba - Univap, E-mail: caroll.guimaraes@yahoo.com.br.

2 Doutoranda em Engenharia Biomédica, Universidade do Vale do Paraíba - Univap, E-mail: apaula@outlook.com.br.

3 Mestrando em Física e Astronomia, Universidade do Vale do Paraíba - Univap, E-mail: gimeneshumberto@outlook.com.

${ }^{4}$ Doutora em Geofísica, Universidade do Vale do Paraíba - Univap, E-mail: virginia@univap.br.

5 Doutora em Engenharia Biomédica, Universidade do Vale do Paraíba - UNIVAP, E-mail: fpupio@univap.br.

6 Human Movement and Rehabilitation (PPGMHR), University Center of Anápolis (UniEVANGÉLICA), Anápolis, GO, Brazil / Programa de Pós-Graduação em Bioengenharia - Laboratório de Biofotônica e Terapêutica Experimental em Saúde e Estética - Universidade Brasil, E-mail: ralopesmartins@gmail.com.

${ }^{7}$ Doutor em Engenharia Biomédica, Universidade do Vale do Paraíba - UNIVAP, E-mail: mol@univap.br.
} 
Palavras-chave: Doenças neurológicas. Reabilitação cardiorrespiratória. Reabilitação sensório-motora. Laserterapia de baixa intensidade. Laserterapia transcraniana.

Abstract: Neurological diseases are usually associated with important functional deficits, making it difficult to perform activities of daily living and self-care. Recent research demonstrates positive effects of Transcranial Photobiomodulation (T-PBM) for the stimulation of the brain tissue. The study aimed to evaluate the effects of T-PBM associated with the aerobic training on the function of the musculoskeletal system of individuals with chronic neurological injuries. In this double-blind clinical trial, 16 volunteers participated with different CNS lesions, of both sexes, with an average age of $51.9 \pm 13.9$ years and preserved gait. The volunteers were randomly divided into 2 groups (aerobic training + active T-PBM and aerobic training + placebo T-PBM). The rehabilitation program was performed on a treadmill, twice a week and with a total duration of 12 weeks. T-PBM ( 3 lasers at $\lambda=660 \mathrm{~nm}$ and 3 lasers at $\lambda=808 \mathrm{~nm}$, $100 \mathrm{~mW}, 6 \mathrm{~J} /$ laser, 60 sec/point, $36 \mathrm{~J}$ per point, total energy $108 \mathrm{~J} / \mathrm{cm} 2$ ) was applied at 3 points, namely Fz, F7 and F8, according to the International 10-20 EEG System. The RMS of the paretic femoral rectus muscle or of a greater impairment in the pretreatment were evaluated, at the 8th and 12th weeks. There was an increase in the electrical activity of the Laser group in the isotonic and isometric RMS collections, and a reduction in muscle recruitment in orthostatism when the beginning and end of the training are compared. It is suggested that T-PBM may have been an influential factor for the increase in muscle recruitment in the Laser group.

Keywords: Neurological diseases. Cardiorespiratory rehabilitation. Sensory motor rehabilitation. Low level laser therapy. Transcranial laser therapy.

Data de submissão: 23.03 .2020

Data de aprovação: 10.05 .2021

Identificação e disponibilidade:

(https://revista.univap.br/index.php/revistaunivap/article/view/2492, http://dx.doi.org/10.18066/revistaunivap.v27i56.2492.

\section{INTRODUÇÃO}

Alterações no desenvolvimento e maturação do tecido nervoso, traumas de diferentes tipos e interrupções do aporte sanguíneo podem levar a importantes lesões neurológicas, como no caso da Paralisia Cerebral (PC), Lesão Medular (LM), Traumatismo Crânioencefálico (TCE) e Acidente Vascular Encefálico (AVE), respectivamente. As consequências clínicas decorrentes do dano ao tecido nervoso variam de acordo com o tipo, local e extensão da lesão, gerando na maioria das vezes incapacidades residuais funcionais, sensoriais, comportamentais e/ou cognitivas permanentes (FURLAN; FEHLINGS, 2008; PIASSAROLI et al., 2012; BILLINGER et al., 2012; O'LEARY; NICHOL, 2018; EDWARDS, 2019).

A principal característica após lesão do neurônio superior é chamada de espasticidade, sendo descrita por um aumento do tônus basal devido à alteração na modulação dos sinais das vias corticoespinhais e da diminuição ou perda das influências inibitórias (BIERING-SORENSEN; NIELSEN; KINGLER, 2006; BORELLA; 
SACCHELI., 2009; AZE et al., 2016). Em longo prazo a espasticidade estimula a remodelação do tecido muscular - do fenótipo de fibras e suas proporções, principalmente em grandes musculaturas, reduz significativamente o número de unidades contráteis, dificulta a seletividade muscular, reduz a capacidade oxidativa e aumenta o gasto energético para realização de movimentos dinâmicos, impactando o desempenho de atividades de vida diária e auto-cuidado (TEIXEIRA-SALMELA et al., 2000; SEVERINSEN et al., 2016; SYNNOT et al., 2017; ENGLISH et al.,2010).

Exercícios aeróbicos realizados em esteira permitem um estímulo mais intenso ao sistema cardiovascular, na tentativa de impulsionar o processo de reabilitação e estimular o condicionamento físico de pacientes com lesões neurológicas (LEE et al., 2008; COMBS-MILLER et al., 2014). Além disso, sua prática regular parece estar ligada a melhora da função cortical, mediada pelo aumento do fluxo sanguíneo e liberação de mediadores químicos e neurotróficos, importantes para processos de angiogênese, neurogênese e neuroplasticidade (ANTUNES et al., 2006; HUANG et al., 2014; Ll et al., 2014; FALCK et al., 2019).

A aplicabilidade da Terapia Laser de Baixa Intensidade (TLBI) ou Fotobiomodulação (FBM), como é conhecida atualmente, tem se mostrado efetiva dentro do processo terapêutico para objetivos que variam desde cicatrização de feridas, alívio de dor crônica e processos inflamatórios, diminuição da fadiga, diminuição de marcadores bioquímicos pós exercícios à melhora do desempenho muscular de indivíduos sadios e neurológicos (LEAL JUNIOR et al., 2010; DE MARCHI et al., 2011; ALMEIDA et al., 2011;CHAIEB et al., 2015; REIS et al., 2015; NAMPO et al., 2016; MONICH; BAVRINA; MALINOVSKAYA, 2018; NEVES et al., 2020).

Estudos apontam que a FBM pode ser considerada uma importante ferramenta para estimulação do tecido nervoso e um potencial facilitador para recuperação em diferentes condições neurológicas (LAMPL et al., 2007; ZIVIN et al., 2009; SCHIFFER et al., 2009; NAESER et al., 2011). Estes benefícios podem ser a atribuídos a melhora de oxigenação de áreas cerebrais lesionadas, favorecendo a ação mitocondrial, a modulação da excitabilidade cortical e de fatores inflamatórios, a produção energética e liberação de fatores neurotróficos, importantes para a neuroplasticidade (NEASER et al., 2011; CASSANO et al., 2015). Para tal efeito indica-se a aplicação diretamente sobre a cabeça, em locais onde não há presença de cabelos (HENNESSY; HAMBLIN, 2017).

Sendo assim, a combinação entre os efeitos benéficos do exercício aeróbico e da FBM-T poderia potencializar a ativação cortical, para secundariamente, aumentar o desempenho muscular de indivíduos neurologicamente acometidos. Portanto, este estudo teve como objetivo avaliar os efeitos da terapia combinada (FBM-T e exercício em esteira) sobre o sistema músculoesquelético de indivíduos com sequelas crônicas de Lesão do Sistema Nervoso Central (SNC).

\section{METODOLOGIA}

Trata-se de um estudo experimental transversal, randomizado, controlado por placebo. Este estudo foi aprovado pelo Comitê de Ética e Pesquisa (CEP) da Universidade do Vale do Paraíba (UNIVAP) sob o protocolo CAAE: 94858718.3.0000.5503, e conta com registro no Clinical Trials $\mathrm{N}^{\circ}$ NCT03751306. Todos os voluntários recrutados faziam parte da lista de espera da clínica de fisioterapia da UNIVAP e do cadastro de pacientes que não se encontravam em atendimento. $O$ estudo foi realizado na clínica de Fisioterapia da Faculdade de Ciências da Saúde (FCS) da Universidade do Vale do Paraíba, em conjunto com o 
Laboratório de Engenharia de Reabilitação Sensório Motora pertencente ao Instituto de Pesquisa e Desenvolvimento (IP\&D).

Para serem considerados elegíveis os voluntários deveriam ter diagnóstico de doenças neurológicas crônicas (a partir de 6 meses) de Acidente Vascular Encefálico de origem isquêmica (AVEi), Traumatismo Crânioencefálico (TCE), Lesão Medular (LM), Paralisia Cerebral (PC), pós-operatório de tumor cerebral ou Esclerose Múltipla (EM), ter entre 18 e 85 anos, cognição preservada e capacidade de responder a estímulos verbais, marcha preservada com ou sem dispositivo de auxílio, regularidade no uso de medicamentos contínuos e liberação médica para a reabilitação. Foram excluídos tabagistas ativos, portadores de patologias respiratórias crônicas e doenças cardíacas descompensadas, obesidade grau II ou III, pacientes com lesão medular acima de T6 e que apresentassem disreflexia autonômica ou com escalas ASIAA e B, pacientes com Esclerose Múltipla em período de surto, uso de fármacos betabloqueadores e AVE hemorrágico.

\subsection{AMOSTRA}

Inicialmente 20 indivíduos se encaixavam nos critérios de inclusão para participação do estudo, porém 4 voluntários foram afastados por problemas de saúde. Os voluntários considerados aptos foram distribuídos aleatoriamente nos grupos Laser ou Placebo por critério de sorteio realizado por um segundo pesquisador. Para garantir o design duplo-cego do estudo, este mesmo pesquisador foi responsável pela aplicação da FBM-T. Além disso, o tratamento placebo foi projetado para ser estruturalmente equivalente, fornecendo condições semelhantes ao dos voluntários pertencentes ao grupo Laser. Cada grupo foi composto por 8 participantes, sendo estes: Grupo Laser: reabilitação sensório-motora associada à aplicação ativa da FBMT; Grupo Placebo: reabilitação sensório-motora associada à aplicação placebo da FBM-T.

\subsection{AVALIAÇÃO CLÍNICA}

Foi avaliada a atividade elétrica do músculo Reto Femoral parético ou de maior comprometimento em 3 momentos: (A0) Pré-tratamento; (A1): Aproximadamente na $8^{\mathrm{a}}$ semana de reabilitação; (A2): Aproximadamente na $12^{\mathrm{a}}$ semana, correspondente ao final da reabilitação.

Para captação do sinal eletromiográfico (EMG) utilizou-se o aparelho da marca EMG System do Brasil Ltda, modelo EMG832 C - USB, de oito canais, composto por um conversor A/D (conversor analógico-digital) de 12 bits de resolução, acoplados a um computador. A calibração ocorreu de acordo com especificações do fabricante, com frequência de amostragem de ordem de $2000 \mathrm{~Hz}$. Para fixação dos eletrodos levou-se em consideração a recomendação de Surface Electromyography for the NonInvasive Assessment of Muscles (SENIAM) e prova de função muscular do lado parético.

Após a delimitação da área, realizou-se tricotomia seguida de higienização com álcool $70 \%$. Eletrodos adesivos descartáveis foram posicionados no sentido das fibras musculares, na região mais proeminente do ventre muscular. Para identificação do sinal de referência, utilizou-se um eletrodo em forma de pinça sobre o processo estilóide da ulna, longe do eletrodo ativo.

Todos os voluntários foram instruídos a ficarem em pé, com os pés levemente afastados, mãos apoiadas em um espaldar e olhar fixo no horizonte. As coletas 
aconteceram a partir de três situações distintas: (A) manutenção do ortostatismo durante 15 segundos; $(B)$ durante o movimento de agachamento a $45^{\circ}$ segundo comando do terapeuta, pelo máximo de repetições completas em 20 segundos; (C) isometria do movimento de agachamento em 15 segundos. Os dados respectivos ao sinal da EMG de superfície foram arquivados em forma de TXT e transportados posteriormente para um software de extração do valor de Root Mean Square (RMS).

O processamento dos valores de RMS foi realizado pelo software ELEDA®, acrônimo de Electromyography and Dynamometer Data Analysis, desenvolvido por pesquisadores do laboratório de Física e Astronomia, pertencentes ao IP\&D da Universidade do Vale do Paraíba, São José dos Campos. Todos os dados da amplitude do sinal foram normalizados pelo software entre 1 e -1 . A fim de padronizar a extração de RMS, cortes de tamanhos similares foram feitos nos espectros da coleta em ortostatismo e isométrica. Já na coleta isotônica, foram selecionadas 3 contrações completas e semelhantes entre si para obtenção de um valor médio. Todas as janelas de contração foram selecionadas visivelmente.

\subsection{FOTOBIOMODULAÇÃOTRANSCRANIANA}

Um cluster de laser (Modelo Med Light Laser VIV, DMC, Brasil), composto de 6 lasers foi utilizado na tentativa de estimular o aumento do fluxo sanguíneo das artérias cerebral anterior e média. A delimitação das áreas de aplicação foi baseada no Sistema Internacional 10-20 de EEG, sobre os pontos Fz, F7 e F8 respectivamente. Durante a aplicação da FBM-T, somente o pesquisador responsável pela aplicação ficava no ambiente. Como medida de segurança, o mesmo utilizava óculos de proteção adequados ao comprimento de onda escolhido.

Todos os pacientes utilizavam óculos com bloqueio total da visão para que não soubessem da randomização dos grupos. Uma distância mínima foi mantida entre o equipamento e a pele dos voluntários para que evitar possíveis sensações dérmicas de formigamento, agulhamento ou aquecimento que permitissem a descoberta de sua distribuição entre os grupos de tratamento.

Tabela 1 - Característica do equipamento Cluster e parâmetros de irradiação.

\begin{tabular}{cc}
\hline N lasers CLUSTER & 6 \\
\hline Comprimento de onda & (3) $660 \mathrm{~nm}-$ vermelho \\
& $(3) 808 \mathrm{~nm}-$ infravermelho \\
Frequência & Contínua \\
Potência & $100 \mathrm{~mW}$ \\
Energia por laser & $6 \mathrm{~J}$ \\
Tempo de aplicação & $60 \mathrm{~s}$ \\
Número de áreas irradiadas & 3 \\
Energia por ponto de aplicação & $36 \mathrm{~J} / \mathrm{cm}^{2}$ \\
Energia total & $108 \mathrm{~J} / \mathrm{cm}^{2}$ \\
Modo de aplicação & Pontual, mais próximo ao tecido, porém sem \\
& contato, com ângulo de $90^{\circ}$ \\
\hline
\end{tabular}

Fonte: Elaborado pelos Autores. Dados da pesquisa (2020). 
Figura 1 - Posicionamento do voluntário para aplicação da FBM-T e delimitação da área de irradiação do laser.

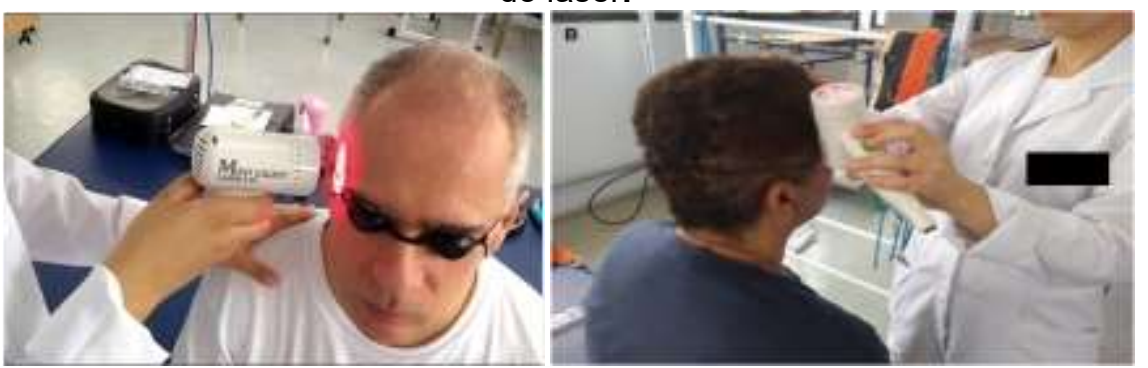

Legenda: Posicionamento do cluster de laser no grupo ativo (à direita) e no grupo placebo (à esquerda)

Fonte: Elaborado pelos Autores. Dados da pesquisa (2020).

\section{PROTOCOLO DE TRATAMENTO}

Cada participante realizou 24 sessões de treinamento em esteira. A reabilitação teve duração de 12 semanas, na frequência de 2 vezes por semana, em dias alternados e com duração de aproximadamente 1 hora.

O protocolo completo consistiu em 10 minutos de repouso ao chegarem ao local dentro do qual recebiam a aplicação ativa ou simulação da FBM-T, 5 minutos de aquecimento em velocidade baixa, 15 minutos de resistência em intensidade moderada ( $50-70 \%$ da frequência cardíaca de treinamento determinada pela equação de Karvonen após teste ergométrico precedente), 5 minutos de desaquecimento com redução gradual da velocidade e 5 minutos de descanso final.

Entre todas as fases eram coletados os sinais vitais como: pressão arterial sistêmica (PAS), frequência cardíaca ( $F C)$, saturação periférica de oxigênio $\left(\mathrm{SatO}_{2}\right)$ e o valor da escala de percepção de esforço (BORG), que deveria ser mantida durante o treinamento entre os valores de 12 a 14 (percepção de esforço moderado).

Os voluntários que não conseguiam realizar o protocolo completo tiveram o treinamento dividido em 2 ou 3 blocos menores de tempo, variando de acordo com índice BORG e com o intervalo de descanso entre as séries. Assistência física foi fornecida apenas quando necessária como forma de garantir a continuidade dos passos, além de estímulos verbais para manutenção do ritmo de marcha.

Todas as fases do final treinamento ocorreram na esteira ergométrica (Moviment RT200 ${ }^{\circledR}$ ), utilizando o apoio da Estação de Reabilitação e Atividade Física BrainMov ${ }^{\circledR}$ para segurança em caso de desequilíbrio ou queda.

Figura 2 - Estação de Reabilitação e Atividade Física BrainMov ${ }^{\circledR}$ utilizada durante o protocolo de reabilitação aeróbica (à esquerda) e posicionamento do voluntário durante a sessão (à direita).
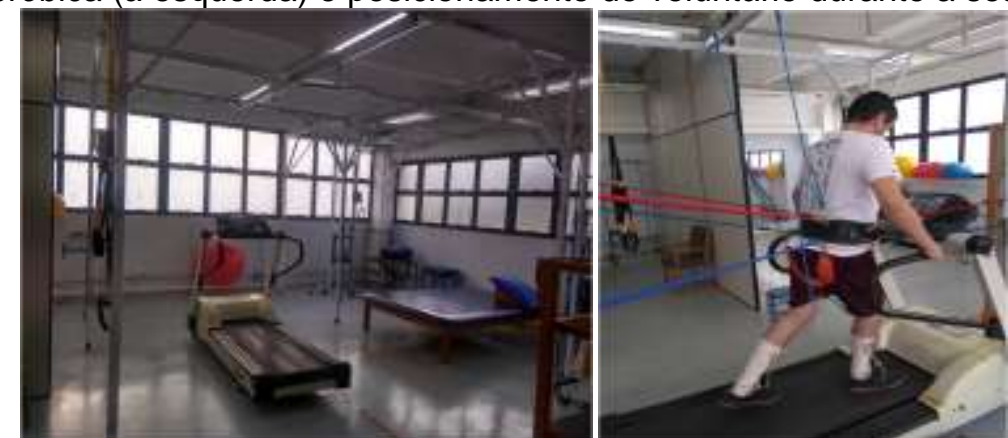

Fonte: Elaborado pelos Autores. Dados da pesquisa (2020). 
A etapa de fortalecimento muscular compreendia dois exercícios voltados para ativação das musculaturas envolvidas na marcha: (A) flexão de quadril e joelho em cadeia cinética aberta, tanto do lado parético como do lado sadio; (B) agachamento. Foram realizadas 3 séries com 10 repetições cada. Conforme evolução, cargas externas (caneleiras) eram adicionadas para resistência ao movimento de cadeia cinética aberta.

Figura 3 - Demonstrativo do exercício de fortalecimento realizado após o protocolo de reabilitação cardiorrespiratório.

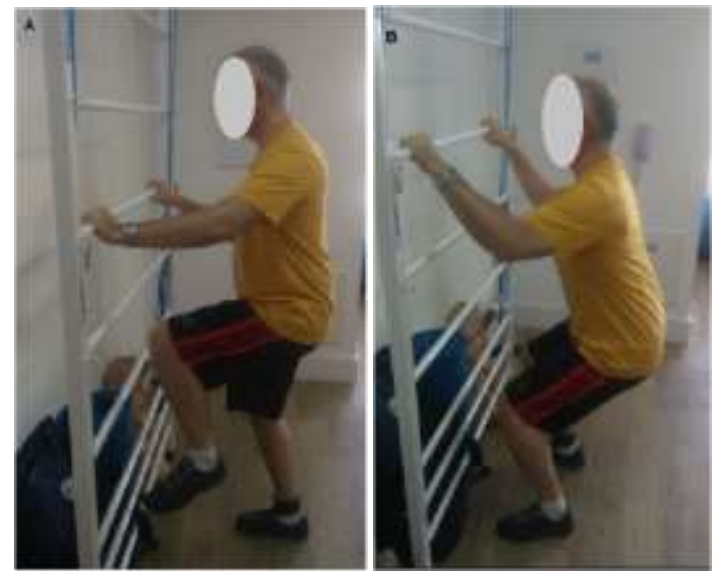

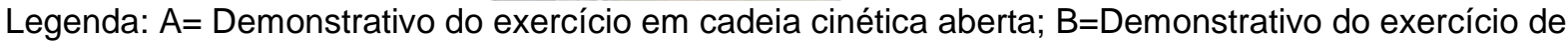
agachamento.

Fonte: Elaborado pelos Autores. Dados da pesquisa (2020).

\section{ANÁLISE DOS DADOS}

Os dados referentes à eletromiografia de superfície coletados durante as avaliações foram organizados e tabulados em forma de planilha no Microsoft Office Excel $^{\circledR} 2013$, apresentando análise descritiva inicial por meio de tendência central e desvio padrão. Toda a análise estatística foi realizada com a ajuda do programa Past ${ }^{\circledR}$ versão 4.0. O teste de Shapiro-Wilk foi aplicado para verificar a normalidade dos dados. Para as amostras consideradas normais, utilizou-se o teste estatístico tstudent, e para as amostras consideradas não normais utilizou-se o teste Wilcoxon. Para verificar a existência de diferenças estatísticas entre as médias de dados (grupo [laser $x$ placebo] $x$ tipo de coleta $x$ tempo de avaliação [pré-tratamento $x 8$ semanas $x$ 12 semanas]) utilizou-se ANOVA, com pós teste Tukey. $O$ valor de alfa foi ajustado para um nível de significância de $p<0,05$.

\section{RESULTADOS}

Dezesseis indivíduos participaram do estudo (10 indivíduos do gênero masculino

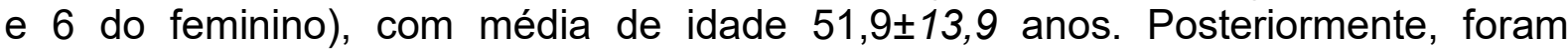
aleatoriamente divididos em 2 grupos: Laser e placebo. A tabela 2 traz um demonstrativo da composição e características de cada grupo. 
Tabela 2 - Caracterização dos grupos Laser e Placebo.

\begin{tabular}{ccc}
\hline CARACTERÍSTICAS & GRUPO LASER & GRUPO PLACEBO \\
\hline$N^{\circ}$ & 8 & 8 \\
Idade & $46,6 \pm 13,8$ & $57,3 \pm 11,7$ \\
Peso & $73,3 \pm 22,2$ & $75,5 \pm 12,4$ \\
Altura & $161,4 \pm 10$ & $169,9 \pm 8,5$ \\
Gênero & $62,5 \% \mathrm{~F}$ & $12,5 \% \mathrm{~F}$ \\
Tipos de lesões & $37,5 \% \mathrm{M}$ & $87,5 \% \mathrm{M}$ \\
Tempo médio de & AVE $(5) ; \mathrm{TCE}(1) ;$ & AVE (5); TCE (1); PC (1); LM \\
acometimento & $\mathrm{PC}(2)$ & $(1)$ \\
\hline
\end{tabular}

Legenda: Média \pm Desvio padrão; F=Feminino; M=Masculino; AVE (Acidente Vascular Encefálico); TCE

(Traumatismo Crânioencefálico); PC (Paralisia Cerebral); LM (Lesão Medular).

Fonte: Elaborado pelos Autores. Dados da pesquisa (2020).

O estudo foi projetado para ser clinicamente similar. Por isso, caso não fosse possível ao participante realizar o treino completo em esteira, o mesmo poderia ser dividido em blocos menores variando de acordo com o tempo de descanso e o índice BORG. Do grupo Laser, 4 participantes tiveram o treinamento dividido em blocos, necessitando de auxílio para manutenção dos passos, enquanto 3 indivíduos do grupo placebo precisaram do mesmo amparo.

As análises do sinal EMG respectivos ao RMS em uV do músculo reto femoral parético nas posturas ortostática, durante o agachamento isotônico e isométrico, expressos como média e desvio padrão (DP) para os grupos Laser e Placebo estão descritos na tabela 3.

Tabela 3 - Valores normalizados de RMS do músculo Reto femoral parético coletados em ortostatismo (repouso), isotonia e isometria do movimento de agachamento durante as avaliação prétratamento, 8 semanas e 12 semanas para os Grupos Laser e Placebo.

\begin{tabular}{|c|c|c|c|c|c|c|}
\hline \multirow{2}{*}{ COLETA } & \multicolumn{2}{|c|}{ PRÉ-TRATAMENTO } & \multicolumn{2}{|c|}{8 SEMANAS } & \multicolumn{2}{|c|}{12 SEMANAS } \\
\hline & G. Las & G. Pla & G. Las & G. Pla & G. Las & G. Pla \\
\hline Ortostatismo & $0,28 \pm 0,04$ & $0,23 \pm 0,02$ & $0,25 \pm 0,03$ & $0,27 \pm 0,03$ & $0,24 \pm 0,04$ & $0,26 \pm 0,05$ \\
\hline Isotônico & $0,17 \pm 0,05$ & $0,19 \pm 0,04$ & $0,22 \pm 0,05$ & $0,19 \pm 0,03$ & $0,20 \pm 0,05$ & $0,19 \pm 0,04$ \\
\hline Isométrico & $0,22 \pm 0,04$ & $0,23 \pm 0,03$ & $0,23 \pm 0,06$ & $0,26 \pm 0,03$ & $0,25 \pm 0,02$ & $0,25 \pm 0,02$ \\
\hline \multirow{2}{*}{\multicolumn{7}{|c|}{ 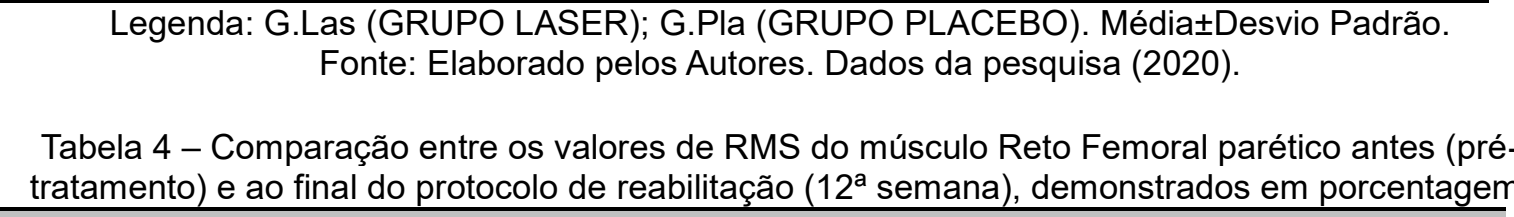 }} \\
\hline & & & & & & \\
\hline \multicolumn{2}{|c|}{ COLETA } & \multicolumn{3}{|c|}{ Grupo Laser (\%) } & \multicolumn{2}{|c|}{ Grupo Placebo (\%) } \\
\hline \multicolumn{2}{|c|}{ Ortostatismo } & \multicolumn{3}{|c|}{$-14,3$} & \multicolumn{2}{|c|}{13} \\
\hline \multicolumn{2}{|c|}{ Isotônico } & \multicolumn{3}{|c|}{17,6} & \multicolumn{2}{|l|}{0} \\
\hline \multicolumn{2}{|c|}{ Isométrico } & \multicolumn{3}{|c|}{13,6} & \multicolumn{2}{|c|}{8,9} \\
\hline
\end{tabular}

Fonte: Elaborado pelos Autores. Dados da pesquisa (2020). 
Diferenças significativas foram encontradas na comparação entre as coletas realizadas em ortostatismo pré-tratamento $\times 8$ semanas para o grupo Placebo $(p 0,03)$ e isotônica no período 8 semanas $\times 12$ semanas $(p 0,04)$ para o grupo Laser. Na comparação entre grupos (Laser vs Placebo), somente a avaliação pré-tratamento realizada em ortostatismo mostrou-se estatisticamente diferente $(p 0,04)$. Não foram encontradas diferenças significativas nas demais comparações intra e entre grupos para os diferentes tipos e tempo de avaliação.

Figura 4 - Representação do sinal de RMS (uV) do músculo reto femoral parético coletado em ortostatismo (repouso) nos períodos de avaliações pré-tratamento, 8 semanas e 12 semanas para os grupos Laser e Placebo.

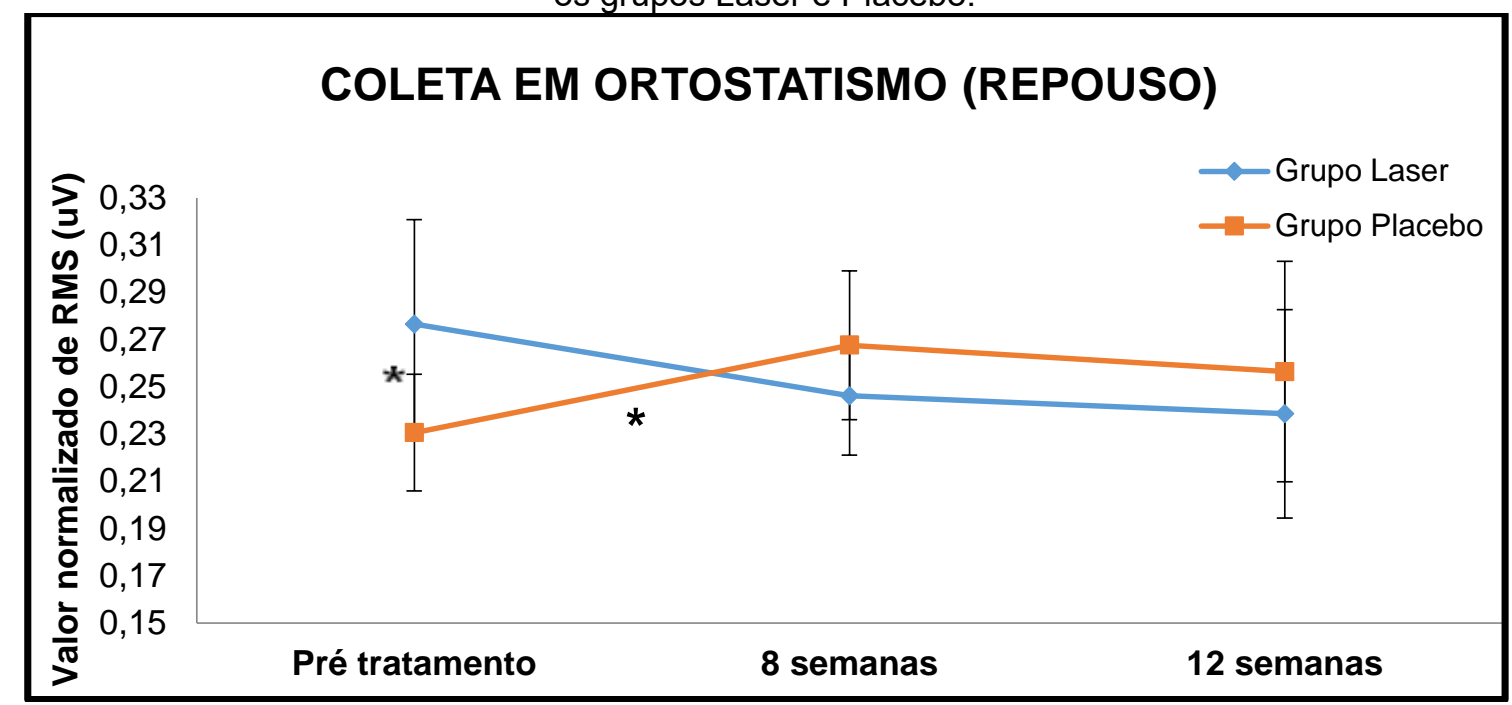

Legenda: Valores representados pela média e as barras de erro representam o desvio padrão. * $p<0,05$.

Fonte: Elaborado pelos Autores. Dados da pesquisa (2020).

Figura 5 - Representação do sinal de RMS (uV)do músculo reto femoral parético coletado durante o movimento isotônico de agachamentonos períodos de avaliações pré-tratamento, 8 semanas e 12 semanas para os grupos Laser e Placebo.

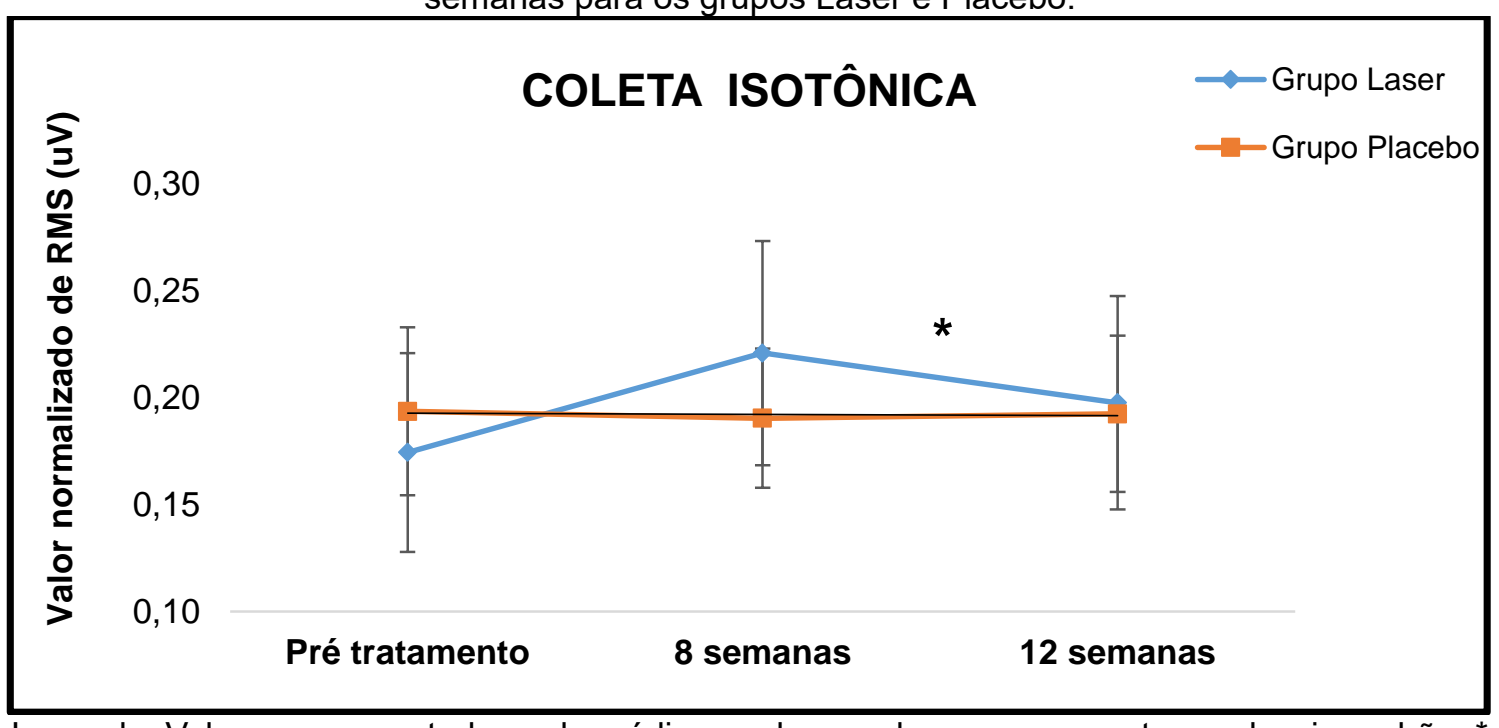

Legenda: Valores representados pela média e as barras de erro representam o desvio padrão. $p<0,05$.

Fonte: Elaborado pelos Autores. Dados da pesquisa (2020). 
Figura 6 - de avaliações pré-tratamento, 8 semanas e 12 semanas para os grupos Laser e Representação do sinal normalizado de RMS (uV)do músculo reto femoral parético coletados durante o movimento isométrico de agachamento nos períodos Placebo.

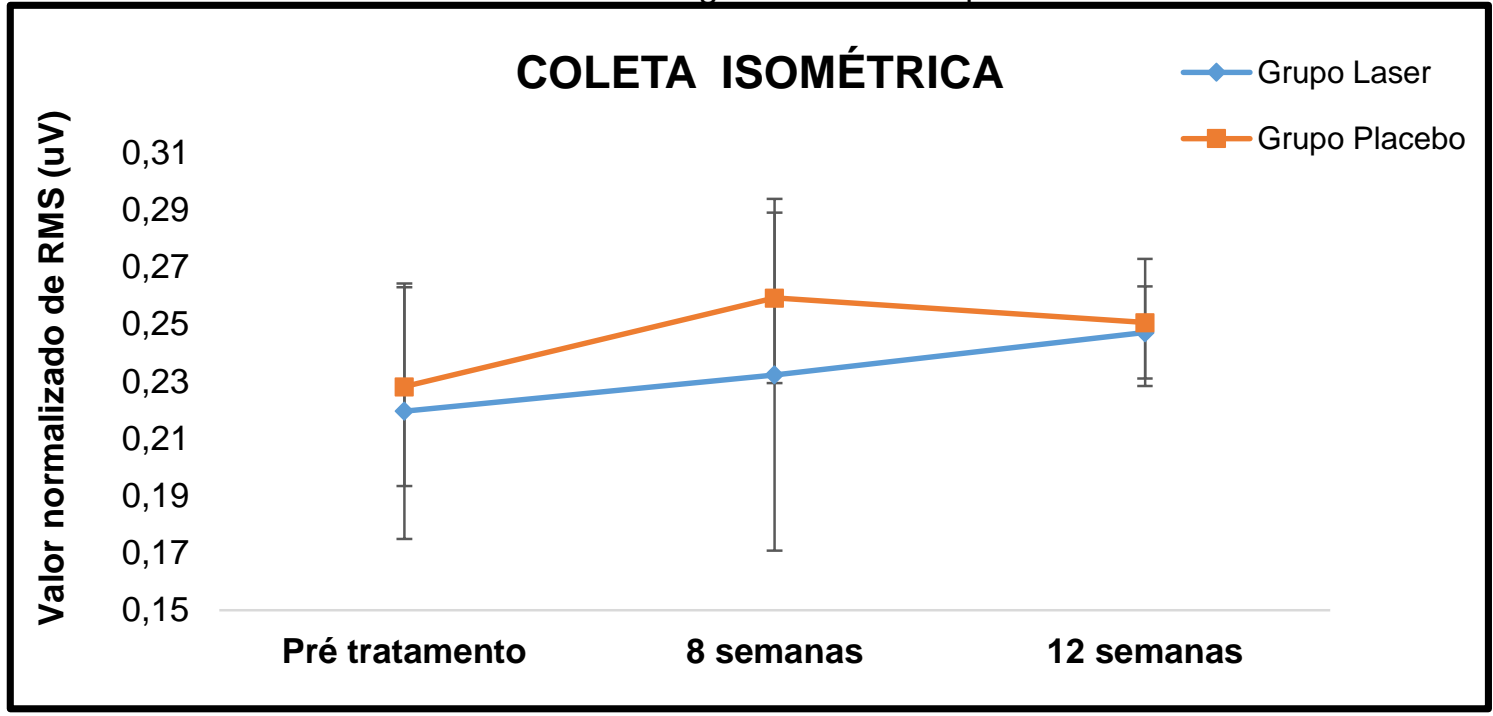

Legenda: Valores representados pela média e as barras de erro representam o desvio padrão. * $p<0,05$.

Fonte: Elaborado pelos Autores. Dados da pesquisa (2020).

\section{DISCUSSÃO}

Apesar da vasta literatura disponível acerca da aplicabilidade laser em diferentes doenças neurológicas (LAMPL et al., 2007; ZIVIN et al., 2009; SCHIFFER et al., 2009; NAESER et al., 2011; ROJAS; BRUCHEY;GONZALEZ-LIMA., 2012; OUESLATI et al., 2015; DISNER; BEEVERS, GONZALEZ-LIMA, 2016; TIAN et al., 2016; VARGAS et al., 2017; HIPSKIND et al., 2018; ZINCHENKO et al., 2019; CHAN et al., 2019), nenhum estudo correlacionando a FBM-T a um possível aumento da atividade muscular periférica foi encontrado tratando-se, portanto, de estudo pioneiro e de grande relevância.

Quanto à eletromiografia músculo reto femoral espástico ou de maior comprometimento, observou-se uma redução de 14,8\% em relação à amplitude do sinal coletado durante a manutenção do ortostatismo entre as avaliações prétratamento e final (12 semanas) para o grupo Laser, o que poderia sugerir uma modulação temporária da hiperatividade muscular. O inverso foi percebido nas demais coletas para ambos os grupos, porém em maior proporção para o grupo Laser. Detectou-se um aumento do recrutamento de fibras de $17,6 \%$ para a contração isotônica e de $13,6 \%$ para a contração isométrica nos indivíduos do grupo Laser. Diferenças significativas foram encontradas na comparação entre as coletas ortostatismo pré-tratamento $\times 8$ semanas para o grupo Placebo $(p 0,03)$ e isotônica no período 8 semanas $\times 12$ semanas ( 0,04$)$ para o grupo Laser. Na comparação entre grupos (Laser vs Placebo), somente a avaliação pré-tratamento realizada em ortostatismo se mostrou estatisticamente diferente $(p 0,04)$. Não foram encontradas diferenças estatísticas entre as médias de dados pelo teste ANOVA (grupo [laser $x$ placebo] $\mathrm{x}$ tipo de coleta $\mathrm{x}$ tempo de avaliação [pré-tratamento $\times 8$ semanas $\mathrm{x} 12$ semanas]).

Um estudo realizado por Konstantinovic e colaboradores (2013) demonstrou que a FBM-T foi capaz de modular a excitabilidade do córtex motor em 14 indivíduos saudáveis. Para tal avaliação, mediu-se a amplitude dos potenciais evocados motores 
(MEPs) do músculo interósseo da mão direita após irradiação transcraniana sobre o córtex motor primário $\left(\lambda 905 \mathrm{~nm}, \mathrm{DP}=50 \mathrm{~mW} / \mathrm{cm}^{2}\right.$, energia total $\left.=15 \mathrm{~J} / \mathrm{cm}^{2}, T=60 \mathrm{~s}\right)$. As mensurações dos MEPs foram feitas antes e após a estimulação laser (até 30 minutos) por meio da estimulação magnética transcraniana. Como resultado, notouse queda na amplitude do MEPs comparados ao baseline, com diferença significativa em 10min (T0-T10 p=0,023) e 20min (T0-T20 p=0,003). Não houve diferença na comparação T0-T30 ( $p=0,18)$. O estudo de Chaieb e colaboradores (2015) corroboram com os resultados supracitados. Também se avaliou a excitabilidade cortical por meio da medição do MEP do músculo interósseo após irradiação transcraniana $(\lambda 810 \mathrm{~nm}$, $P=0,15 \mathrm{~W}, \mathrm{DP}=$ próximo a $\left.500 \mathrm{~mW} / \mathrm{cm}^{2}, \mathrm{DE}=257,14 \mathrm{~J} / \mathrm{cm}^{2} \mathrm{~A}=0,35 \mathrm{~cm}^{2}, \mathrm{~T}=600 \mathrm{~s}, \mathrm{E}=90 \mathrm{~J}\right)$. Houve diminuição de $20-30 \%$ da amplitude dos MEPs imediatamente após a irradiação e até o trigésimo minuto $(p<0,005)$ para o grupo Laser, associado a um aumento da inibição cortical de curto intervalo.

Segundo estes autores, os resultados podem ser atribuídos a estimulação de vias inibitórias, aumentando a neurotransmissão GABAérgica e/ou diminuindo ações Glutamatérgicas. Outro mecanismo citado é a possível alteração do potencial de membrana. Um aumento da atividade da bomba de sódio geraria uma estabilidade membranar e resistência a despolarização, diminuindo a propagação do impulso nervoso.

A especificidade é um princípio fundamental do treinamento físico, tendo como bases o volume, intensidade, frequência de treinamento e tipo de atividade para promover uma resposta adaptativa. Este contexto é essencial para desenvolvimento de força, massa e/ou resistência muscular (COFFEY et al., 2016). Exercícios aeróbicos realizados em alta intensidade seriam capazes de promover aumentos significativos na circulação sérica de biomarcadores como o fator neurotrófico devirado do cérebro (BDNF) e fator de crescimento endotelial vascular (VEGF), importantes para a neuroplasticidade central, além de estarem associados a um equilíbrio da excitabilidade corticoespinhal, o que permitiria maior ativação motora central em indivíduos neurologicamente acometidos (BOYNE et al., 2019; BOYNE et al., 2020).

O treinamento de marcha realizado em esteira permite maior intensidade de exercício quando comparado a reabilitação convencional, chegando a um número 10 vezes maior de passos (HESSE; WERNER; BYHAHN, 2015). Munari e colaboradores (2018), relatam que treinamento em esteira contribui para um equilíbrio na geração de torque dos músculos quadríceps e isquiotibiais paréticos vs lado não parético, promovendo uma maior eficiência biomecânica da marcha.

Nosso estudo sugere que a FBM-T promoveu um efeito modulatório temporário, alterando a excitabilidade dos neurônios do trato corticoespinhal em sincronia com o aumento da influência inibitória. A associação com o treinamento aeróbico pode ter resultado em um aumento do recrutamento de fibras pela possível ativação das vias alfa, relacionadas à contração voluntária. Contudo, novos estudos clínicos são necessários para confirmar esta hipótese.

Não foram encontradas diferenças significativas na funcionalidade dos indivíduos a partir da aplicação da FBM-T. Devemos considerar, portanto, duas possibilidades. A primeira trata-se da cronicidade das lesões e das alterações músculoesqueléticas já instaladas, necessitando talvez de um tempo maior de tratamento para que possíveis mudanças morfofuncionais fossem alcançadas. A segunda poderia estar relacionada ao cálculo de energia, o local de aplicação para estímulo do tecido biológico e a variável do distanciamento da aplicação, não permitindo o cálculo dos parâmetros de densidade de potência e densidade de 
energia.

A grande variabilidade na escolha de parâmetros como tipo de luz, comprimento de onda, tempo de irradiação, potência e energia podem dificultar a modulação do laser e levar a diferentes resultados (SALGADO et al., 2014). Hamblin (2016) relata a importância de considerar o objetivo e a área a ser estimulada para que a escolha dos parâmetros seja clinicamente eficaz para estimulação do tecido biológico.

Características anatômicas e variações de idade também podem influenciar na penetração e interação da luz com tecido. Um estudo realizado por Yuan e colaboradores (2020) avaliou o comportamento de diferentes comprimentos de onda entre faixas etárias que variavam entre 5 e 89 anos. Cinco comprimentos de onda foram utilizados $(680 \mathrm{~nm}, 810 \mathrm{~nm}, 850 \mathrm{~nm}, 980 \mathrm{~nm}$ e $1064 \mathrm{~nm})$ para estimulação das áreas F3-F4 e Fp1-Fpz-Fp2 (córtex pré-frontal dorsolateral e córtex pré-frontal ventromedial) relevantes para o transtorno depressivo. Observou-se que a penetração da luz no tecido diminui à medida que a idade aumenta, correlacionada ao aumento da espessura dos tecidos extracerebrais (couro cabeludo e crânio) e resistência vascular. Os resultados deste estudo demonstram a importância do planejamento do tratamento e da personalização dos parâmetros de acordo com as características de cada indivíduo.

As disfunções neurológicas geralmente se relacionam a importantes déficits funcionais, com impacto econômico significativo para as políticas de saúde pública. A heterogeneidade dos sinais clínicos e de sua intensidade dificultam a criação de uma abordagem unicamente efetiva. Uma terapia não invasiva, segura e relativamente de baixo custo como a FBM, utilizada em sinergismo com o processo de neurorreabilitação poderia otimizar a recuperação motora de pacientes neurológicos crônicas.

\section{CONCLUSÃO}

Diante dos achados encontrados neste estudo, pode-se sugerir que a FBM-T modulou temporariamente a hiperatividade muscular em repouso e, aumentou em maior proporção o recrutamento de fibras do músculo reto femoral parético durante as coletas eletromiográficas isotônicas e isométricas do grupo Laser ao final das 12 semanas de treinamento sensório-motor, apesar de não haver diferenças significativas entre os grupos.

Os resultados deste trabalho são promissores e abrem caminhos para o desenvolvimento de novas pesquisas para investigar a influência da FBM-T no sistema sensório-motor de pacientes com sequelas neurológicas. Acreditamos que a Fotobiomodulação será a estratégia mais promissora para a neurorreabilitação nos próximos anos.

\section{REFERÊNCIAS}

ALMEIDA, P. et al. Red (660 nm) and infrared (830 nm) low-level laser therapy in skeletal muscle fatigue in humans: what is better? Lasers Med. Sci., v. 27, n. 2, p. 453-458, 2011.

ANTUNES, H. K. M. et al. Exercício físico e função cognitiva: uma revisão. Rev. Bras. Med. Esporte, Niterói, v. 12, n. 2, p. 108-114, 2006.

AZE, O. et al. Structural and physiological muscle changes after post-stroke 
hemiplegia: A systematic review. Ann Phys. Rehabil. Med., v. 59, p. e79, 2016.

BIERING-SORENSEN, F.; NIELSEN, J. B.; KINGLER, K. Spasticity-assessment: a review. Spinal Cord., [s.I.], v. 44, p. 708-722, 2006.

BILLINGER, S. A. et al. Reduced Cardiorespiratory Fitness After Stroke: Biological Consequences and Exercise-Induced Adaptations. Stroke Res Treat., v. 2012, p. 111, 2012.

BORELLA, M. P.; SACCHELLI, T. Os efeitos da prática de atividades motoras sobre a neuroplasticidade. Rev. Neurocienc., v. 17, n. 2, p. 61-9, 2009.

BOYNE, P. et al. Exercise intensity affects acute neurotrophic and neurophysiological responses post-stroke. J. Appl. Physiol., v. 126, p. 431-443, 20192018.

Doi:10.1152/japplphysiol.00594.2018

BOYNE, P. et al. Effects of Exercise Intensity on Acute Circulating Molecular Responses Poststroke. Neurorehabil. Neural Repair, v. 34, n.3, p. 222-234, 2020. Doi/10.1177/1545968319899915

CASSANO, P. et al. Near-Infrared Transcranial Radiation for Major Depressive Disorder: Proof of concept study. Psychiatry J., v. 2015, 2015.

DOI:10.1155/2015/352979

CHAIEB, L. et al. Neuroplastic effects of transcranial near-infrared stimulation (tNIRS) on the motor cortex. Front. Behav. Neurosci., v. 9, 2015. DOI: 10.3389/fnbeh.2015.00147

CHAN, A. S. et al. Photobiomodulation Improves the Frontal Cognitive Function of Older Adults. J. Geriatr. Psychiatry, v. 34, n. 2, p. 369-377, 2019.

COFFEY, V. G. et al. Concurrent exercise training: do opposites distract? J. Physiol., v. 595 , n. 9 , p. $2883-2896,2016$

COMBS-MILLER, S. A. et al. Body weight-supported treadmill training vs. overground walking training for persons with chronic stroke: a pilot randomized controlled Trial. Clin. Rehabil., v. 28, n. 9, p. 873-884, 2014.

DE MARCHI, T. et al. Low-level laser therapy (LLLT) in human progressive-intensity running: effects on exercise performance, skeletal muscle status, and oxidative stress. Lasers Med. Sci., v. 27, n. 2, p. 453-8, 2012.

DISNER, S. G; BEEVERS, C. G.; GONZALEZ-LIMA, F. Transcranial Laser Stimulation as Neuroenhancement for Attention Bias Modification in Adults with Elevated Depression Symptoms. Bain Stimul., v. 9, n. 5, p. 780-787, 2016.

EDWARDS, J. D. Cardiovascular disease and other childhood-onset chronic conditions in adults with cerebral palsy. Dev. Med. Child Neurol., v. 61, p. 859-860, 2019. 
ENGLISH, C. et al. Loss of skeletal muscle mass after stroke: a systematic review. Int. J. Stroke, v. 5, 395-402, 2010. DOI: 10.1111/j.1747-4949.2010.00467.x

FALCK, R. S. et al. Impact of exercise training on physical and cognitive function among older adults: a systematic review and meta-analysis. Neurobiol. Aging., v. 79 , p. 119-130, 2019.

FURLAN, J. C.; FEHLINGS, M. G. Cardiovascular complications after acute spinal cord injury: pathophysiology, diagnosis, and management. Neurosurg. Focus, v. 25, n. 5: E13, 2008.

HAMBLIN, M. R. Shining light on the head: Photobiomodulation for brain disorders. BBA Clin., v. 6, p. 113-124, 2016

HENNESSY, M.; HAMBLIN, M. R. Photobiomodulation and the brain: a new paradigm. J. Opt., v. 19, n. 1, 2017.

HESSE, S.; WERNER, C.; BYHAHN, M. Transcranial low-level laser therapy may improve alertness and awareness in traumatic brain injured subjects with severe disorders of consciousness: A case series. Int. Arch. Med., v. 8, n. 144, p. 1-12, 2015.

HIPSKIND, S. G. et al. Pulsed Transcranial Red/Near-Infrared Light Therapy Using Light-Emitting Diodes Improves Cerebral Blood Flow and Cognitive Function in Veterans with Chronic Traumatic Brain Injury: A Case Series. Photomed. Laser Surg., v. 37, n. 2, p. 77-84, 2018.

HUANG, T. et al. The effects of physical activity and exercise on brain-derived neurotrophic factor in healthy humans: A review. Scand. J. Med. Sci. Sports, v. 24, p. 1-10, 2014.

KONSTANTINOVIC, L. M. et al. Transcranial application of near-infrared low-level laser can modulate cortical excitability. Lasers Surg. Med., n. 45, p. 648-653, 2013.

LAMPL, Y. et al. Infrared Laser Therapy for Ischemic Stroke: A New Treatment Strategy: Results of the NeuroThera Effectiveness and SafetyTrial-1 (NEST-1). Stroke, v. 38, n.6, p. 1843-1849, 2007.

LEAL JUNIOR, E. C. et al. Effects of low-level laser therapy (LLLT) in the development of exercise-induced skeletal muscle fatigue and changes in biochemical markers related to post exercise recovery. J. Orthop. Sports Phys. Ther., v. 40, n. 8, p. 524-32, 2010.

LEE, M. J. et al. Comparison of Effect of Aerobic Cycle Training and Progressive Resistance Training on Walking Ability After Stroke: A Randomized Sham ExerciseControlled Study. J. Am. Geriatr. Soc., v. 56, n. 6, p. 976-985, 2008.

LI, L. et al. Acute Aerobic Exercise Increases Cortical Activity during Working Memory: A Functional MRI Study in Female College Students. PLoSOne, v. 9, n. 6, 
e99222, 2014.

MONICH, V. A.; BAVRINA, A. P.; MALINOVSKAYA, S. L. Modification in oxidative processes in muscle tissues exposed to laser- and light-emitting diode radiation. Lasers Med. Sci., v. 33, n. 1, p. 159-164, 2018.

MUNARI, D. et al. High-intensity treadmill training improves gait ability, VO2peak and cost of walking in stroke survivors: preliminary results of a pilot randomized controlled trial. Eur. J. Phys. Rehabil. Med., v. 54, n. 33, 408-418, 2018.

NAESER, M. A. et al. Improved Cognitive Function After Transcranial, Light-Emitting Diode Treatments in Chronic Traumatic Brain Injury: Two Case Reports. Photomed Laser Surg., v. 29, n. 5, p. 351-358, 2011.

NAMPO, F. K. et al. Low-level phototherapy to improve exercise capacity and muscle performance: a systematic review and meta-analysis. Lasers Med. Sci., v. 31, n. 9, p. 1957-1970, 2016.

NEVES, M. F. et al. Long-term analyses of spastic muscle behavior in chronic poststroke patients after near-infrared low-level laser therapy $(808 \mathrm{~nm})$ : a doubleblinded placebo-controlled clinical trial. Lasers Med. Sci., v. 35, p. 1459-1467, 2020. https://doi.org/10.1007/s10103-019-02920-3

O'LEARY, R. A.; NICHOL, A. D. Pathophysiology of severe traumatic brain injury. J. Neurosurg. Sci., v. 62, n. 5, p. 542-548, 2018.

OUESLATI, A. et al. Photobiomodulation suppress alpha-synuclein-induced toxicity in an AAV-based rat genetic model of Parkinson's disease. PLoS One, v. 10, n. 10, p. Pe0140880, 2015. DOI: 10.1371/journal.pone.0140880.

PIASSAROLI, C. A. P. et al. Modelos de Reabilitação Fisioterápica em Pacientes Adultos com sequelas de AVE isquêmico. Rev. Neurocienc., v. 20, n. 1, p. 128-137, 2012.

REIS, M. C. et al. Immediate effects of low-intensity laser $(808 \mathrm{~nm})$ on fatigue and strength of spastic muscle. Lasers Med. Sci., v. 30, n. 3, p. 1089-1096, 2015.

ROJAS, J. C.; BRUCHEY, A. K.; GONZALEZ-LIMA, F. Low-level light therapy improves cortical metabolic capacity and memory retention. J. Alzheimers Dis., v. 32, n. 3, p. 741-752, 2012.

SALGADO, A. S. I. et al. The effects of transcranial LED therapy (TCLT) on cerebral blood flow in the elderly women. Lasers Med. Sci., v. 30, n. 1, p. 339-346, 2014.

SCHIFFER, F. et al. Psychological benefits 2 and 4 weeks after a single treatment with near infrared light to the forehead: a pilot study of 10 patients with major depression and anxiety. Behav. Brain Funct., v. 5, n. 46, p. 1-13, 2009.

SEVERINSEN, K. et al. Skeletal muscle fiber characteristics and oxidative capacity in hemiparetic stroke survivors. Muscle Nerve, v. 53, n. 5, p. 748-54, 2016. 
SYNNOT, A. et al. Interventions for managing skeletal muscle spasticity following traumatic brain injury. Cochrane Database Syst. Rev., v.11, n.11, p. CD008929, 2017. doi: 10.1002/14651858.CD008929.pub2..

TEIXEIRA-SALMELA, L. F. et al. Fortalecimento muscular e condicionamento físico em hemiplégicos. Acta Fisiátrica, v. 7, n. 3, p. 108-118; 2000.

TIAN, F. et al. Transcranial laser stimulation improves human cerebral oxygenation. Lasers surg. med., v. 48, p. 343-349, 2016.

VARGAS, E. et al. Beneficial neurocognitive effects of transcranial laser in older adults. Lasers in Medical Science, v. 32, n. 5, p. 1153-1162, 2017.

YUAN, Y. et al. Transcranial photobiomodulation with near-infrared light from childhood to elderliness: simulation of dosimetry. Neurophotonics, V.7, n.1, p.015009-015009, 2020.

ZINCHENKO, E. et al. Pilot study of transcranial photobiomodulation of lymphatic clearance of beta-amyloid from the mouse brain: breakthrough strategies for nonpharmacologic therapy of alzheimer's disease. Biomed. Opt. Express, v. 10, n. 8, p. 4003-4017, 2019.

ZIVIN, J. A. et al. Effectiveness and Safety of Transcranial Laser Therapy for Acute Ischemic Stroke (NEST-2). Stroke, v. 40, p. 1359-1364, 2009. 